\title{
Philosophiques
}

\section{Figures de l'empirisme}

\section{François Duchesneau}

Volume 12, numéro 2, automne 1985

URI : https://id.erudit.org/iderudit/014433ar

DOI : https://doi.org/10.7202/014433ar

Aller au sommaire du numéro

Éditeur(s)

Société de philosophie du Québec

ISSN

0316-2923 (imprimé)

1492-1391 (numérique)

Découvrir la revue

Citer ce document

Duchesneau, F. (1985). Figures de l'empirisme. Philosophiques, 12(2), 412-420.

https://doi.org/10.7202/014433ar

Ce document est protégé par la loi sur le droit d'auteur. L'utilisation des services d'Érudit (y compris la reproduction) est assujettie à sa politique d'utilisation que vous pouvez consulter en ligne.

https://apropos.erudit.org/fr/usagers/politique-dutilisation/
Cet article est diffusé et préservé par Érudit.

Érudit est un consortium interuniversitaire sans but lucratif composé de l’Université de Montréal, l’Université Laval et l’Université du Québec à Montréal. Il a pour mission la promotion et la valorisation de la recherche. https://www.erudit.org/fr/ 


\section{FIGURES DE L'EMPIRISME par François Duchesneau}

Permettez-moi d'abord une brève citation : «Si nous avions été capables de prendre toujours la nature pour guide, nous saurions tout, en quelque sorte, sans avoir rien appris. C'est qu'elle ne prend pas le ton des philosophes, qui, lors même qu'ils nous égarent, ne cessent de nous traiter d'ignorants. " ${ }^{1}$

C'est en reprenant à mon compte ces propos quelque peu démystificateurs d'un philosophe français, expert en analyse, que j'exprimerai à la Société Royale du Canada ma reconnaissance pour l'accueil qu'elle m'a réservé parmi ses membres. Je dois à la vérité de dire que j'aspire à être très peu philosophe pour ne pas être de ces philosophes qui tout en nous égarant, s'emploient à nous traiter d'ignorants. De longue date, je me suis exercé à cet empirisme qui consiste à ouvrir les yeux pour recueillir la leçon de ce que la nature nous fait voir. Je ne sais donc si je puis m'autoriser du titre de philosophe, mais je voudrais vous exposer certaines raisons de mon allégeance à l'empirisme, cette philosophie si peu philosophique. Comme l'empirisme n'a pas de contours dogmatiques, je tenterai d'en redessiner pour vous quelques figures, quelques modèles d'accomplissement, qui motivent mes recherches et dont je suis heureux de reconnaître qu'ils ne sont nullement ma propriété exclusive.

La destinée de l'empirisme est d'avoir parti lié avec la science et ses pratiques de connaissance. Ainsi, à partir du siècle de Périclès, les auteurs de la Collection hippocratique développèrentils des modèles de telles pratiques qui allaient motiver par la suite la théorie de la connaissance des sceptiques grecs et alexandrins. La méthodologie d'observation, celle qui a fait la juste réputation d'Hippocrate et de ses premiers disciples, ne se détachait pas d'une doctrine de l'ordre des phénomènes ; mais l'observation se présentait en fait comme le révélateur de cet ordre ; et sur l'observation systématisée devait se construire l'édifice d'une pratique rationnelle. Si certains hippocratiques conjuguent leur conception de la nature à des hypothèses atomistes sur la structure des corps vivants et non-vivants et sur les méca-

1. Condillac, La langue des calculs, Texte établi et présenté par Anne-Marie Chouillet. Introduction et notes de Sylvain Auroux, Lille : Presses Universitaires de Lille, 1981, p. 9. 
nismes d'interaction de ces corps, ils réduisent ces hypothèses atomistes à un rôle auxiliaire et ils ploient la signification des concepts théoriques pour leur faire épouser les contours des phénomènes physiologiques et pathologiques. Lorsque quelques siècles plus tard, Sextus Empiricus s'en prend aux paradoxes des théories rationalistes de la connaissance, c'est le modèle hippocratique qui lui sert d'argument, et c'est l'empirisme sous cette forme qu'il entend promouvoir par la critique des positions dogmatiques et spéculatives des Stoïciens. Mais l'Antiquité n'est pas mon domaine de recherche. Je me tourne donc d'emblée vers la genèse de l'empirisme moderne.

Sans doute des philosophies empiristes accompagnent-elles les moments principaux dans l'avènement de la science moderne. $\mathrm{Au} 17^{\mathrm{e}}$ siècle, Francis Bacon est un témoin privilégié de cette histoire, tout comme Hobbes et Gassendi donnant la réplique au rationaliste Descartes. Mais la science nouvelle est partagée entre deux types de pratique discursive. D'une part, elle tend à se structurer suivant le modèle des démonstrations de géométrie ; d'autre part, elle reprend la recette de l'empirisme antique en se fixant comme idéal de décrire et de classer les phénomènes à partir du châtoiement multiple des propriétés sensibles. La science moderne connaît ses premières réalisations significatives lorsque la pratique rationnelle tente de concilier ces méthodes antagonistes. Et la vieille hypothèse atomiste des démocritéens ressurgit alors comme garant d'une représentation de l'ordre naturel permettant de telles audaces : la conciliation s'opère entre la raison géométricienne, qui reconstruit déductivement le mécanisme des phénomènes, et le jugement d'expérience qui poursuit sans limite préétablie l'inventaire factuel de la nature. L'antinomie est trop simple pour rendre compte seule du développement concret des sciences. Mais il reste que l'histoire de la science moderne s'inscrit sous les instances conflictuelles des méthodes géométrique et observationnelle, en même temps qu'un principe heuristique en constitue la trame de fond : le mécanisme corpusculaire comme doctrine de l'ordre naturel.

C'est à la fin du $17^{\mathrm{e}}$ siècle, à l'époque de John Locke et d'Isaac Newton que se produit la mutation profonde d'où surgissent à la fois une science de la nature émancipée de l'inter- 
rogation métaphysique et, conjointement, une théorie empiriste de la connaissance prenant la science en voie de développement pour objet privilégié d'analyse. À l'aube du siècle des Lumières, l'œuvre de science commence donc à se doubler d'une œuvre de critique de la science. Le premier grand livre de la philosophie des Lumières est sans doute l'Essay concerning Human Understanding (1690) de Locke. Dans l'Épître au lecteur, Locke nous informe que l'entreprise de construction de la science possède désormais ses propres maîtres d'œuvre, ses "master-builders", et il donne l'exemple de Boyle, Huygens, Sydenham et Newton. Le philosophe conserve pour sa part un rôle de subalterne, "underlabourer" : ce rôle consiste à débarrasser le terrain sur lequel l'édifice se construit, de tout ce qui peut entraver la bonne marche des travaux. L'objet plus positif de la philosophie est de formuler une théorie de la connaissance qui établisse la légitimité du savoir scientifique en développement, la limitation de ses objectifs, car nous ne voulons pas d'une nouvelle Tour de Babel métaphysique, et la validité des méthodes mises en œuvre. Le philosophe ne décrète plus ce que doit être la science ; il dresse l'inventaire factuel et critique des profits et pertes d'une entreprise dont il vérifie les comptes. Dans mes premières recherches, celles qui aboutirent à mon travail sur L'empirisme de Locke, ${ }^{2}$ je m'interrogeais sur les conditions déterminantes et les formes de cette mutation lockienne de l'épistémologie. L'idée se développait en moi que la volonté d'harmoniser la "plain, historical method" des baconiens et l'hypothèse corpusculaire de Boyle, de dépasser les présupposés métaphysiques sous-tendant l'empirisme de Gassendi, de concevoir la construction géométrique comme justifiée a posteriori conformément à la pratique de Huygens, l'idée se développait en moi que cette volonté s'était coulée dans le moule d'une méthodologie de traitement des phénomènes, celle que l'on trouve illustrée dans la pathologie empiriste de Sydenham. Les textes de méthodologie médicale, sans doute écrits en collaboration par Sydenham le praticien et Locke l'épistémologue, me servaient de fil d'Ariane pour réinterpréter les articulations majeures de la dérivation empirique des idées complexes, les normes, délimitations et modes de construction

2. F. Duchesneau, L'empirisme de Locke, La Haye : Martinus Nijhoff, 1973. 
du savoir et de la probabilité. Je parvenais à mettre en valeur l'idée d'une architectonique immanente à ce déploiement empirique des connaissances, architectonique ayant de profondes racines dans une conception « hippocratique -» de l'ordre naturel.

Le fait suivant auquel je ferai référence, tient à la célèbre formule que Newton insère dans le scolie général ajouté à la $3^{\mathrm{e}}$ partie dans la seconde édition des Philosopbice naturalis principia mathematica (1713) : "Hypotheses non fingo ". Il s'agissait pour Newton d'éviter la critique lancinante des disciples de Descartes, critique qui hante la conscience même de Newton héritier de la physique cartésienne : l'explication du théorème de la gravitation est incomplète si l'on ne fournit une explication de la force gravitationnelle suivant les concepts d'une mécanique de l'impulsion par contact. Et hélas, de ce point de vue cartésien, l'explication semble vouée à l'incomplétude ! Il faut tuer le père ou du moins faire comme si on l'avait tué. Newton commet ce parricide, ou fait semblant de le commettre, en suspendant la justification ontologique des concepts les plus fondamentaux de la théorie. La science sera justifiée par sa progression méthodique, par la rigueur et l'ampleur de sa mainmise sur l'expérience. L'édifice n'a plus besoin d'être rationnellement fondé dans ses postulats ultimes. Le palais du savoir scientifique peut être construit sur le sable, contrairement à ce que pensait Descartes, car les matériaux utilisés et les recettes d'utilisation permettent de prendre appui légèrement sur la surface du sol ; en outre, l'édifice a une propension à s'étirer suivant l'horizontale en épousant les sinuosités du terrain plutôt que de s'ériger à la verticale d'un seul tenant en forme définitive. Newton, le savant par excellence, a refusé de se soumettre aux normes de la rationalité métaphysique. Ce faisant, il propose au professionnel de la philosophie l'œuvre de science comme un paradoxe épistémologique à analyser et dénouer si possible. Cette autonomie nouvellement proclamée de la science par rapport à la philosophie constitue le fait primordial pour ce qui est des formes de la connaissance scientifique au $18^{\mathrm{e}}$ siècle.

Cette figure de l'empirisme j'ai tenté de la redessiner pour les théories physiologiques : d'où l'ensemble des travaux qui ont abouti à mon livre La Physiologie des Lumières. Empirisme, modèles 
et théories. ${ }^{3}$ Il appartient aux institutions de la science de faire cohabiter des constellations divergentes constituées d'éléments conceptuels et méthodologiques. Le disparate n'exclut pas pour autant une rationalité des programmes de recherche, aptes à susciter l'innovation théorique et expérimentale et les transformations successives d'une constellation donnée. Par ailleurs, les constellations divergentes peuvent même se trouver en opposition très prononcée. Jamais l'antithèse n'est alors intégrale, à moins qu'on ne prenne le caractère contradictoire de certains présupposés de part et d'autre pour une condition d'incompatibilité radicale. Certes, le philosophe, réfléchissant sur les structures formelles du discours scientifique, a tendance à traduire les constellations dont nous venons de parler, en strictes constructions hypothético-déductives, et alors les conditions logiques de l'incompatibilité se dévoilent au regard. Mais si l'on a une conception moins rigide, moins formaliste, de la rationalité en contexte de découverte, il devient apparent que la science se développe en s'açcommodant d'incohérences relatives ou plus exactement en tentant de les surmonter à la longue. Dans de nombreux cas, la coexistence de programmes de recherche en divergence initiale engendre divers modes d'intersection des constellations qui évoluent en se faisant face.

Ainsi se développe au $18^{\mathrm{e}}$ siècle l'opposition des options mécanistes et vitalistes dans les théories physiologiques et les méthodologies d'étude sur l'être vivant. Une hypothèse que je ne développerai pas, mais qu'on pourrait étayer d'arguments, est que cette vaste antinomie, à l'œuvre dans la théorisation biologique depuis le $18^{\mathrm{e}}$ siècle, est encore au cœur de la biologie et de la biophilosophie aujourd'hui : d'où quelques lignes directrices pour une recherche à venir en philosophie de la biologie contemporaine,

Le $17^{\mathrm{e}}$ siècle finissant avait légué aux physiologies mécanistes de Boerhaave, de Baglivi, de Hoffmann des processus d'analyse visant à repérer les micro-structures emboịtées ou juxtaposées en lesquelles peut se détailler la structure des organes : il s'agit d'inférer de l'anatomie subtile, dont Malpighi avait

3. F. Duchesneau, La Pbysiologie des Lumières. Empirisme, modèles et théories, La Haye, Boston, Londreș : Martinụs Nijhoff, 1982. 
fourni le modèle, une exacte conception des micro-structures, de leurs opérations et des fonctions résultantes au niveau des organes. L'effort pour rendre compte de telles opérations repose sur le recours à des analogies tirées conjointement d'actes fonctionnels conscients et d'effets physiques explicables par masse, figure, impulsion, mais aussi, suivant le modèle newtonien, par des forces spécifiques d'attraction ou de répulsion. Il est évident que c'est une combinaison des analogies psychologiques et physiques qui semble rendre compte de l'autorégulation, de l'intégration et de l'adaptabilité des processus vitaux. Le programme de type mécaniste cesse d'être essentiellement spéculatif, lorsque Haller au milieu du siècle publie ses Elementa physiologia corporis bumani (1757-1766). Haller conjugue les éléments suivants : 1) les principes d'une méthodologie newtonienne appliquée aux phénomènes complexes ; 2) une théorie unifiée des éléments de structure organique : les fibres de diverses sortes ; 3 ) le repérage des phénomènes spécifiques à ces divers ordres de fibres ; 4) l'identification de propriétés fonctionnelles élémentaires : l'irritabilité et la sensibilité ; et 5) l'hypothèse d'une intégration par paliers des structures organiques sous un plan architectonique incarné dans les dispositifs préformés de l'embryon : ces dispositifs préformés gouverneraient les mutations organiques et fonctionnelles subséquentes. Bien entendu, Haller attribue aux propriétés fonctionnelles des fibres élémentaires un statut analogue à celui des forces d'attraction s'exerçant entre les parties de la matière non-vivante. $\grave{A}$ travers de multiples transformations, la structure théorique fixée par Haller et son modèle méthodologique domineront l'activité de recherche jusqu'à ce qu'on en vienne à transcrire les opérations vitales en processus physico-chimiques dépendant de structures complexes au début du $19^{\mathrm{e}}$ siècle. Parmi les mutations les plus significatives du programme hallérien, il faut signaler l'abandon du préformationisme structural au profit de conceptions épigénétiques sur la formation des organismes. Une force spécifique conçue suivant l'analogie d'un principe newtonien va même incarner sous forme dynamique le projet architectonique et l'on aura le Bildungstrieb, la vis formativa de Blumenbach. À ce moment précis, la physiologie mécaniste a intégré une forme particulière de la tradition vitaliste issue de Stahl. L'organicisme résultant consiste à conce- 
voir un projet de structuration initiale de l'organisme comme incarné dans une force. Celle-ci s'exercerait lorsque les conditions matérielles appropriées d'organisation se trouvent réunies. On conçoit qu'un tel programme mixte puisse permettre, ce qu'il a fait, d'analyser les conditions organiques des fonctions vitales, tout en ayant recours à des concepts théoriques de type téléologique qui ont pour objet de préciser la spécificité de l'organisme comme système de déterminations matérielles vitales. Ces concepts théoriques promeuvent l'analyse, la classification et l'interprétation des phénomènes par delà le strict point de vue de la micro-anatomie.

Certes, j'ai insisté sur l'antinomie vitalisme/mécanisme, mais je ne voudrais que vous y voyiez le principe d'une dichotomie radicale des théories de l'être vivant. La stricte opposition du mécanisme et du vitalisme est une invention de philosophes plus métaphysiciens qu'épistémologues. L'induction fondée sur l'étude des textes nous suggère plutôt qu'il existe une tension interne dominant l'heuristique des diverses théories concernées. L'analyse descriptive et explicative des fonctions globales représentant l'intégration des processus vitaux, d'une part, est en état de tension par rapport à l'analyse des micro-structures et de leurs phénomènes spécifiques, d'autre part. Le propre de la recherche dans le domaine biologique au $18^{\mathrm{e}}$ siècle est d'abord d'avoir tenté de surmonter par des constructions de plus en plus habiles cet hiatus théorique : le problème spéculatif a stimulé ainsi l'invention de concepts programmatiques de la biologie à venir. Ces constructions ont servi à encadrer l'utilisation de moyens divers pour une analyse structuro-fonctionnelle de plus en plus poussée.

Ajouterai-je que si ces traditions de recherche ont pu s'établir et évoluer, c'est sans doute pour une bonne part en raison de l'épistémologie régnante, héritée de Locke, et qui professe la dérivation empirique des contenus de connaissance, voire la dérivation empirique des capacités analytiques de l'entendement humain ? Recueillir la leçon de l'expérience et la mettre en forme rationnelle est sans doute la directive philosophique principale des Lumières. Et c'est pourquoi partant d'un idéal baconien de méthodologie descriptive l'on passe successivement à des types de plus en plus raffinés de mise en forme rationnelle des données 
d'expérience, les positions significatives de cet empirisme rationnel s'exprimant par exemple chez Condillac, chez Diderot, chez Senebier.

En guise de conclusion ou d'épilogue, j'aimerais attirer votre attention sur quelques aspects de la crise actuelle de la philosophie des sciences, dominée jusqu'à la décennie 60 de notre siècle par l'empirisme logique et par sa contrepartie critique : le falsificationisme de Karl Popper. Dans les deux cas, l'objet privilégié d'analyse épistémologisme était la structure logique d'un discours scientifique reconstruit de façon à permettre d'en contrôler la rigueur formelle et la validité empirique. Contre cette représentation abstraite de la rationalité scientifique, la critique s'est exercée dans les directions les plus diverses. Mais la visée la plus constante nous fixe l'objectif d'une représentation épistémologique qui rende compte plus adéquatement de la science en devenir, de la science dans son histoire concrète. Alors que Reichenbach avait naguère distingué contexte de découverte et contexte de justification en science et rabattu la recherche épistémologique sur le seul contexte de justification, ${ }^{4}$ les tentatives les plus récentes visent au contraire à retracer les processus rationnels de la découverte en y intégrant même les reconstructions justificatives inhérentes à la science en développement. ${ }^{5}$ Suivant la tradition de recherche empiriste, l'histoire des sciences peut ainsi susciter la dérivation des modèles par lesquels sera représentée l'articulation rationnelle de cette histoire. Mais encore là, les méthodes de l'empirisme se déploient entre deux tendances : le scepticisme qui relativise à l'extrême la possibilité de saisir une trame rationnelle dans l'histoire du discours scientifique ; le rationalisme qui tend à normer la progression empirique du savoir suivant des systèmes de représentation, qui correspondent à des vues aprioristes.

Or il y a une leçon des phénomènes qui s'infere empiriquement des phénomènes par séries de constructions a posteriori. La logique elle-même n'est-elle pas un fruit abstrait de cette maturation empirique de la connaissance, sur laquelle j'estime

4. H. Reichenbach, Experience and Prediction, Chicago : University of Chicago Press, 1938.

5. Cf. à titre d'exemple : T. Nickles (ed.), Scientific Discovery, Logic, and Rationality, Dordrecht : D. Reidel, 1980. 
que le philosophe aurait plus que jamais aujourd'hui intérêt à se pencher? Et l'empiriste qui prend en compte le rôle des structures abstraites d'une raison nécessairement a posteriori peut dans une certaine mesure s'accorder à ces propos d'un philosophe allemand, célèbre conciliateur : " Quand cette voie empirique des vérités particulières aurait été l'occasion de toutes les découvertes, elle n'aurait pas été suffisante pour les donner ; et les inventeurs mêmes ont été ravis de remarquer les maximes et les vérités générales quand ils ont pu les atteindre, autrement leurs inventions auraient été fort imparfaites ". ${ }^{6}$ L'empiriste se contenterait sans doute d'ajouter que les maximes et les vérités générales n'ont de sens que dans et par la démarche empirique qui permet de les découvrir en s'en servant.

François Duchesneau, Université de Montréal.

6. Leibniz, Nouveaux essais sur l'entendement, 4.7.11, Paris : Garnier-Flammarion, 1966, p. 367. 